\title{
Submillimeter Spectrum of the Cosmic Background Radiation
}

\author{
Toshio Matsumoto \\ Department of Astrophysics, Nagoya University \\ Chikusa-ku, Nagoya, Japan $46\{-01$
}

Recent observations of the spectrum of the $3 \mathrm{~K}$ cosmic background radiation (CBR) indicate that the CBR spectrum is consistent with a blackbody spectrum of $T=$ $2.74 \pm 0.02 \mathrm{~K}$ (Smoot et al. 1987). These measurements, however, were carried out in the Rayleigh-Jeans part of the spectrum, while theories predict spectral distortion in the Wien part. Therefore, we tried to observe the submillimeter spectrum of the CBR with a liq.He cooled radiometer onboard a sounding rocket. The experiment was a collaboration between Nagoya University and University of California, Berkeley.

The winston light concentrater had a $4 \mathrm{~cm}$ aperture with a $7.6^{\circ}$ beam. A $\ddot{\text { flared }}$ baffle at the top of the horn resulted in very low side lobe response. The photometer was designed to observe sky brightness in 6 filter bands between $100 \mu \mathrm{m}$ and $1 \mathrm{~mm}$ simultaneously (Lange et al. 1987, Sato et al. 1987). Since the whole optical system was cooled down to $1.0 \mathrm{~K}$, instrumental emission was negligible. Careful absolute calibration in the laboratory provided small systematic errors, especially for 3 long wavelength channels.

The sounding rocket, $\mathrm{K}-9 \mathrm{M}-80$, having the radiometer onboard was launched on 1987 February 23, 0:00 JST (February 22, 15:00 UT), from the Kagoshima Space Center of the Institute of Space and Astronautical Science, Japan (Matsumoto et al.1988). The rocket reached an apogee of $317 \mathrm{~km}$ at $287 \mathrm{~s}$ after launch. The lid of the cryostat was opened at $150 \mathrm{~s}$ and the observation commenced. The rocket axis which coincides with the optical axis of the radiometer precessed with a period of $10 \mathrm{~s}$ around $30^{\circ} \pm 1.5^{\circ}$ full cone angle on the sky passing through the zenith. The center of the precession cone pointed towards $l=203^{\circ} \pm 2^{\circ}, b=33^{\circ} \pm 2^{\circ}$. Just after the lid opened, the temperature of the photometer increased a little and environmental emission was observed in the short wavelength channels. After $200 \mathrm{~s}$, however, the transient phenomena disappeared and all signals became steady. Fig.1 shows the average sky brightness of the observed region of the sky.

689

D. McNally (ed.), Highlights of Astronomy, Vol. 8, 689-691.

(C) 1989 by the IAU. 


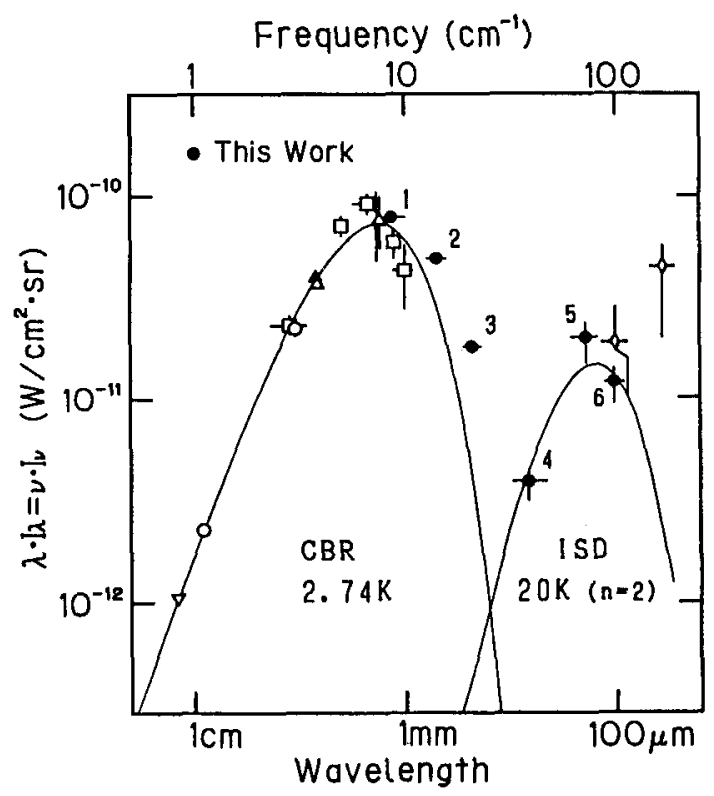

Figure 1: The observed spectrum of the astrophysical background. Filled circles indicate our data. Vertical and horizontal bars indicate $1 \sigma$ errors and bandwidths, respectively.

A characteristic feature of Fig.1 is an excess submillimeter brightness above a blackbody spectrum of $2.74 \mathrm{~K}$. Signals of the three long wavelength channels were spatialy isotropic, indicating their cosmological origin. This is the first significant detection of a distortion of the CBR spectrum in the Wien region.

The spectrum of the three short wavelength channels shows a feature expected for thermal emission of the diffuse interstellar dust (ISD). The spatial distributions of these channels indicate a clear correlation with column density of neutral hydrogen, N(HI), as shown in Fig.2. Assuming that a correlated brightness with N(III) is of galactic origin, then ISD emission was well fit by the thermal emission of the dust, assuming $T_{d}=19 \pm 3 \mathrm{~K}$ and emissivity index of 2 . Ratios of IR-brightness to $\mathrm{N}(\mathrm{HI})$ were also obtained. This ratio at $102 \mu \mathrm{m}, 0.65 \pm 0.14 \mathrm{MJy} \cdot s r^{-1} / 10^{20} \mathrm{H}$, is consistent with that obtained by Terebey and Fich (1986) for IRAS but is a little smaller than that by Boulanger and Perault (1988). The galactic emission thus obtained, however, cannot explain all the observed brightness. In the $102 \mu \mathrm{m}$ band, residual emission could be attributed to zodiacal emission, but a significant isotropic emission $\left(5 \pm 2 \times 10^{-12} W \cdot \mathrm{cm}^{-2} . s r^{-1}\right)$ remains in the $137 \mu \mathrm{m}$ band. The origin of this emission is not certain, but may be related to isotropic emission observed by IRAS at $100 \mu m$ (Boulanger and Perault 1988).

The most important result of this experiment is an excess submillimeter CBR 


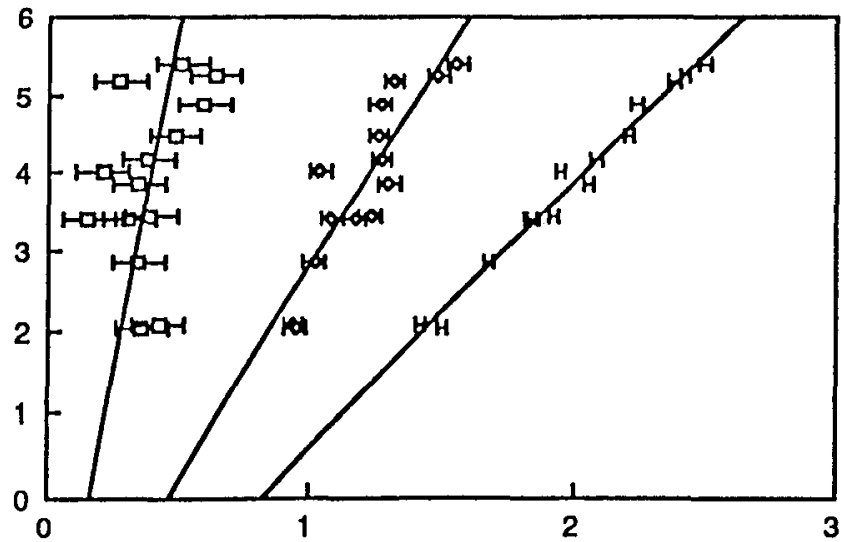

- 102 micron

- 137 micron

a 262 micron

\section{Sub-mm Brightness $/\left(10^{\wedge}-11 \mathrm{~W} / \mathrm{cm}^{\wedge} 2 \mathrm{sr}\right)$}

Figure 2: The correlation of $\mathrm{N}(\mathrm{HI})$ with submillimeter brightness. The horizontal error bars indicate $1 \sigma$ statistical errors only.

brightness. The energy density of this excess amounts to $10 \%$ of a $2.74 \mathrm{~K} \mathrm{CBR}$, which provides a serious restriction on baryonic energy generation at high redshift. Two physical processes which explain the observed spectrum are proposed (Ilayakawa et al. 1987). One is Compton scattering in intergalactic hot plasma. Another is dust emission, which was generated and heated by Pop.III stars in the early universe, superposed on the CBR spectrum. Future advanced studies of the submillimeter CBR will provide a more definite scenario for understanding the origin and evolution of the early universe.

\section{References}

Boulanger,F and Perault,M. 1988, Ap.J., 330, 964.

Hayakawa,S. et al. 1987, Pub.Astr.Soc.Japan, 39, 941.

Lange,A. et al. 1987, Appl.Optics, 26,401.

Matsumoto,T. et al. 1988, Ap.J, 329, 567.

Sato,S. et al. 1987, Appl.Optics, 26, 410.

Smoot,G. et al. 1987, Ap.J.Letters, 317, L45.

Terebey,S and Fich,M 1986, Ap.J.Letters, 309, L73. 